\title{
Local Existence for Boussinesq Equations with Slip Boundary Condition in a Bounded Domain
}

\author{
Jian Lu \\ College of Science, University of Shanghai for Science and Technology, Shanghai, China \\ Email: lujian9702@163.com
}

How to cite this paper: Lu, J. (2017) Local Existence for Boussinesq Equations with Slip Boundary Condition in a Bounded Domain. Journal of Applied Mathematics and Physics, 5, 1951-1963.

https://doi.org/10.4236/jamp.2017.510165

Received: September 13, 2017

Accepted: October 17, 2017

Published: October 20, 2017

Copyright $\odot 2017$ by author and Scientific Research Publishing Inc. This work is licensed under the Creative Commons Attribution International License (CC BY 4.0).

http://creativecommons.org/licenses/by/4.0/

\begin{abstract}
In this paper, we are interested in the local existence for the Boussinesq equations with the slip boundary conditions. Energy method and Gerlakin approach are employed in this paper to get the main result.
\end{abstract}

\section{Keywords}

Boussinesq, Slip Boundary, Local Existence

\section{Introduction}

The Boussinesq equations are as follows:

$$
\left\{\begin{array}{l}
u_{t}+(u \cdot \nabla) u+\nabla p=\gamma \Delta u+\theta f, \\
\theta_{t}+(u \cdot \nabla) \theta=\varepsilon \Delta \theta, \\
\operatorname{divu}=0,
\end{array}(x, t) \in \Omega \times(0, t)\right.
$$

where $\Omega$ is a bounded smooth domain of $R^{3}, \quad u=\left(u^{1}, u^{2}, u^{3}\right)$ and $\theta$ represent density and temperature, $p$ is the pressure function, $f$ is the external force, $\gamma \geq 0, \varepsilon \geq 0$ represent viscous coefficient and thermal conductivity coefficient.

In this paper, the initial data is given by

$$
\left.(u, \theta)\right|_{t=0}=\left(u_{0}, \theta_{0}\right)
$$

and the boundary condition is

$$
\left.u \cdot n\right|_{\partial \Omega}=0,\left.\frac{\partial \theta}{\partial n}\right|_{\partial \Omega}=\alpha \theta,\left.(S u \cdot n)_{\tau}\right|_{\partial \Omega}=(B u)_{\tau}
$$

Boussinesq equations are the classical model of fluid mechanics. There are a lot of important applications in marine ecology and weather forecasting. There are a lot of related conclusions about 2-D Boussinesq equations. Ye Zhuan [1] 
studied the Cauchy problem of the two-dimensional (2D) incompressible Boussinesq equations with fractional dissipation by making use of the nonlinear lower bounds for the fractional Laplacian established in Constantin and Vicol. Xiaojing $\mathrm{Xu}$ [2] studied the Cauchy problem of the two-dimensional (2D) incompressible Boussinesq equations with fractional Laplacian dissipation by the nonlinear lower bounds for the fractional Laplacian. In this paper, we want to study the 3-D Boussinesq equations. On the one hand, the regularity and well-posedness of Boussinesq equations is a popular problem that people study. Jishan fan [3] proved a regularity criterion for the 3D Boussinesq system with partial viscosity. T. Hmidi [4] studied the global well-posedness of the Euler-Boussinesq system with the term dissipation $|D| a$ on the temperature equation. On the other hand, the existence of Boussinesq equations is always an important problem people are interested in. Ying Liu [5] applied the Fourier decomposition method to study the attenuation in $L^{2}$ of the weak existence of the Boussinesq equations. Wei Li [6] used the homogeneous balance method and travelling-wave transformation to acquire some exact solutions of the Boussinesq equations. Xianjin Li [7] studied the global stability in $L^{2}$ of the Boussinesq equations in three dimensional regional and unbounded regional.

However, for the initial questions and the boundary questions, the stations are more complicated and challenging. In fact, in fluid mechanics, two boundary conditions are considered mainly. One is the Diriclet boundary condition: $u=0, x \in \partial \Omega$, and another is the famous boundary condition proposed by Navier:

$$
u \cdot n=0,(S u \cdot n)_{\tau}=-\alpha u_{\tau}, x \in \partial \Omega
$$

where $n$ is the unit outward normal on $\partial \Omega, u_{\tau}$ is the tangential part of $u$, $S u$ denotes the deformation tensor: $S u=\frac{1}{2}\left(\nabla u+\nabla u^{t}\right)$.

Now, what we need to do is the study for the local existence of the problem (1.1) - (1.3). Usually, the followed existences are considered. First, the smooth of the existence is so small [8] [9] [10] that the initial data and the existence are close to a constant in $H^{2}(\Omega)$, however this existence does not have singularity. Second, the existence is the "large energy" proposed by Lions [11]. This existence has regularity but the analysis of the characteristic are more difficult and the uniqueness and the continuous dependence can't be solved [12] [13]. Last, according to documents [14] [15] [16], the initial data in $L^{2}(\Omega)$ is small and the initial density is positive and bounded. For example, when the initial data is piecewise smooth, the solutions shown in [17] satisfy the Rankine-Hugoniot conditions in a strict point wise sense. On the other hand, these solutions have so enough structure and regularity that the uniqueness and continuous dependence theory can be proved in [18]. The well-posedness theory is nearly complete in the whole space, but for the Dirichlet condition: on $\partial \Omega$, the station $u=0$ may have problems. However, if considering the Navier condition described above, the global weak small-energy solutions can be proved to exist in a half space for initial data with small energy and bounded density. David Hoff [19] 
studied the equations

$$
\left\{\begin{array}{l}
\rho_{\mathrm{t}}+\operatorname{div}(\rho u)=0 \\
\left(\rho u^{j}\right)_{t}+\operatorname{div}\left(\rho u^{j} u\right)+P(\rho)_{x_{j}}=\mu \Delta u^{j}+\lambda \operatorname{div}_{x_{j}}+\rho f^{j},
\end{array}\right.
$$

where $\operatorname{div}(\cdot)$ denotes divergence.

Satisfying the bounded conditions:

$$
\left\{\begin{array}{l}
u \cdot v=0, \\
\mu \prod \nabla u v=\prod B u,
\end{array} x \in \Omega, t>0\right.
$$

where $v(x)$ is the unit outward normal on $\partial \Omega, \Pi(x)$ is the projection onto the tangent plant to $\partial \Omega$ at $x$, then the existence of the solutions can be given. $T$. Hmidi [20] studied a fractional diffusion Boussinesq model which couples a Navier-Stokes type equation with fractional diffusion for the velocity and a transport equation for the temperature by establishing global well-posedness results with rough initial data.

In this paper, we consider the Boussinesq equations. Because the temperature and the fluid are coupled together, the study becomes more difficult. The key we solve the problem is how to deal with the temperature.

First, we define a function

$$
W=\left\{w \in\left(H^{1}(\Omega)\right)^{3}: w(x) \cdot n(x)=0, x \in \partial \Omega\right\}
$$

Definition 1.1. For a fixed time $T,(u, \theta)$ is called the solutions for (1.1) - (1.3) on $[0, T]$ if the following holds:

1) $(u, \theta) \in C\left([0, T] ; H^{1}(\Omega)\right) \cap L^{2}\left([0, T] ; W^{2, q}(\Omega)\right)$,

$$
\left(u_{t}, \theta_{t}\right) \in C\left([0, T] ; L^{2}(\Omega)\right) \cap L^{2}\left([0, T] ; H^{1}(\Omega)\right) \text {. }
$$

2) For any times $t_{1}, t_{2} \in[0, T]$,

$$
\begin{gathered}
\int_{t_{1}}^{t_{2}} \int_{\Omega}\left(u_{t}+(u \cdot \nabla) u+\nabla p\right) \varphi \mathrm{d} x \mathrm{~d} t=\int_{t_{1}}^{t_{2}} \int_{\Omega}(\gamma \Delta u+\theta f) \varphi \mathrm{d} x \mathrm{~d} t, \\
\int_{t_{1}}^{t_{2}} \int_{\Omega}\left(\theta_{t}+(u \cdot \nabla) u\right) \phi \mathrm{d} x \mathrm{~d} t=\int_{t_{1}}^{t_{2}} \int_{\Omega}(\varepsilon \Delta \theta) \phi \mathrm{d} x \mathrm{~d} t,
\end{gathered}
$$

for $\varphi, \phi$ Lipschitz $\Omega \times[0, T]$ with $\phi, \varphi \in W$.

Remark: the system parameters $\Omega$ and $B$ will be assumed to satisfy the following conditions:

1) $\Omega$ is a bounded open set in $R^{3}$ with a $C^{3}$ boundary.

2) $B$ is a $C^{1}$ in a neighborhood of $\partial \Omega$.

Theorem 1.2. Assume the hypotheses hold, let $q \in(3,6]$ be fixed constant and the $\left(u_{0}, \theta_{0}\right), p$ and $f$ satisfy the following conditions:

$$
\begin{gathered}
(p, f) \in C\left([0, T] ; L^{2}(\Omega)\right) \cap L^{2}\left([0, T] ; L^{q}(\Omega)\right) ; \\
\left(p_{t}, f_{t}\right) \in L^{2}\left([0, T] ; L^{2}(\Omega)\right) ; \\
\left.(S u \cdot n)_{\tau}\right|_{\partial \Omega}=(B u)_{\tau} .
\end{gathered}
$$

Then there is a small $T_{*}>0$ and a unique strong solution $(u, \theta)$ to the ini- 
tial boundary value problems (1.2), (1.3) such that:

$$
\begin{gathered}
(u, \theta) \in C\left(\left[0, T_{*}\right] ; H^{1}(\Omega)\right) \cap L^{2}\left(\left[0, T_{*}\right] ; W^{2, q}(\Omega)\right) ; \\
\left(u_{t}, \theta_{t}\right) \in C\left(\left[0, T_{*}\right] ; L^{2}(\Omega)\right) \cap L^{2}\left(\left[0, T_{*}\right] ; H^{1}(\Omega)\right) .
\end{gathered}
$$

\section{The Lame Operator and the Regularity of $-\Delta$ Operator}

In this part, we introduce the regularity of Laplace and the $-\Delta$. First we assume the condition (1.2) holds, $\Omega$ and $B$ satisfy the above hypotheses. Considering the following problem: find $u: \bar{\Omega} \rightarrow R^{3}$ such that:

$$
\left\{\begin{array}{l}
L u+\beta u=g, x \in \Omega \\
u \cdot n=0, \\
(S(u) \cdot n)_{\tau}=(B u)_{\tau}, x \in \partial \Omega
\end{array}\right.
$$

here $L u=-\gamma \Delta u$, fixed $\beta \in R$, given $g: \Omega \rightarrow R$. Then the corresponding weak form is achieved.

Use $\omega \in W$ to multiply by the above differential Equation (2.1), we can get:

$$
A_{\beta}(u, \omega)=\int_{\Omega} g \cdot \omega \mathrm{d} x
$$

where $A_{\beta}: W \times W \rightarrow R$ is the bilinear form:

$$
A_{\beta}(u, \omega)=\gamma\left(-\int_{\partial \Omega} A u \cdot \omega \mathrm{d} s+\int_{\Omega} S u: S \omega \mathrm{d} x\right)
$$

Obviously, $A_{\beta}$ is continuous on $W \times W$ if $B$ is bounded, and we can use trace theorem to show that $A_{\beta}$ is coercive if $\beta$ is enough large depending on $\gamma, \Omega, B$. In this case, there is a bounded operator $S_{\beta}: L^{2} \rightarrow W$ satisfying

$$
A_{\beta}\left(S_{\beta} g, \omega\right)=\int_{\Omega} g \cdot \omega \mathrm{d} x
$$

for all $\omega \in W$. Furthermore, because the embedding $W \rightarrow L^{2}$ is compact, $S_{\beta}$ is a compact operator from $L^{2}$ to $L^{2}$ and the symmetry condition guarantees $S_{\beta}$ is self-adjoint.

The following lemmas are taken from document [21].

Lemma 2.1. Assume that $\Omega \subset R^{3}$ is abounded open set with a $C^{3}$ boundary and that $B \in L^{\infty}(\partial \Omega)$ is a symmetric matrix. For $\beta$ enough large, there is a compact self-adjoint operator $S_{\beta}: L^{2}(\Omega) \rightarrow L^{2}(\Omega)$, whose range is contained in $W$ and for which (2.2) holds for $g \in L^{2}(\Omega)$ and $\omega \in W$. At the same time, there is an orthogonal basis $\left\{\omega_{k}\right\}_{k}$ for $L^{2}$ whose elements are in $W \cap C^{\infty}(\Omega)$, and which are eigenfunctions of $S_{\beta}$

$$
S_{\beta} \omega_{k}=\gamma_{k}^{-1} \omega_{k}
$$

where $\gamma_{k} \rightarrow \infty$.

Lemma 2.2. Assume that $m \geq 0, \Omega \subset R^{3}$ is abounded open set with a $C^{m+1}$ boundary and that $B$ is a $C^{m+1}(\partial \Omega)$ mapping from a neighborhood of $\partial \Omega$ into the set of $3 \times 3$ matrices, then there exists a constant $C_{m}=C(\gamma, \Omega, B)$ such that if $u$ is a solution of (2.1) in the sense of (2.2) where $\beta$ and $g \in H^{m}(\Omega)$, then $u \in H^{m+2}(\Omega)$ and 


$$
\|u\|_{H^{m+2}(\Omega)} \leq C_{m}\left(\|u\|_{L^{2}(\Omega)}+\|g\|_{H^{m}(\Omega)}\right)
$$

For the operator $-\Delta$, consider the following problem: find $\theta: \bar{\Omega} \rightarrow R^{3}$ such that

$$
\left\{\begin{array}{l}
-\Delta \theta+\delta \theta=\bar{g}, x \in \Omega \\
\left.\frac{\partial \theta}{\partial n}\right|_{\partial \Omega}=\alpha \theta
\end{array}\right.
$$

where $\delta \in R$ is fixed, $\bar{g}: \Omega \rightarrow R$ is given.

Use $\phi \in H^{1}(\Omega)$ to multiply by the above differential equation, and integration by parts, then we get

$$
B_{\delta}(\theta, \phi)=\int_{\Omega} \bar{g} \cdot \phi \mathrm{d} x
$$

where $B_{\delta}: H^{1} \times H^{1} \rightarrow R$ is the bilinear form:

$$
B_{\delta}(\theta, \phi)=\int_{\Omega} \nabla \theta \cdot \nabla \varphi+\delta \theta \varphi \mathrm{d} x
$$

Similar to the Lame operator, there exist a bounded operator $\Gamma_{\delta}: L^{2}(\Omega) \rightarrow H^{1}$ meeting

$$
B_{\delta}\left(\Gamma_{\delta} g, \phi\right)=\int_{\Omega} \bar{g} \cdot \phi \mathrm{d} x
$$

for all $\varphi \in H^{1}$ and for $\delta$ large enough. Parallel to Lemmas $2.1-2.2$, we can get the following lemmas.

Lemma 2.3. Assume that $\Omega \subset R^{3}$ is abounded open set with a $C^{3}$ boundary. For $\gamma$ large enough, there exist a compact self-adjoint operator $\Gamma_{\gamma}: L^{2}(\Omega) \rightarrow L^{2}(\Omega)$, whose range is contained in $H^{1}$ and for which (2.9) holds for $\bar{g} \in L^{2}(\Omega)$ and $\phi \in H^{1}$. At the same time, there is an orthogonal basis $\left\{\phi_{k}\right\}_{k}$ for $L^{2}$ whose elements are in $H^{1} \cap C^{\infty}(\Omega)$ and which are eigenfunctions of $\Gamma_{\gamma}, \Gamma_{\gamma} \phi_{k}=\overline{\gamma_{k}^{-1}} \omega_{k}$, where $\overline{\gamma_{k}} \rightarrow \infty$.

Lemma 2.4. Assume that $m \geq 0, \Omega \subset R^{3}$ is abounded open set with a $C^{m+1}$ boundary and that $B$ is a $C^{m+1}(\partial \Omega)$ mapping from a neighborhood of $\partial \Omega$ into the set of $3 \times 3$ matrices, then there exists a constant $\overline{C_{m}}=\bar{C}(\Omega)$ such that if $\theta$ is a solution of (2.6) in the sense of (2.7) where $\bar{g} \in H^{m}(\Omega)$, then $\theta \in H^{m+2}(\Omega)$ and

$$
\|\theta\|_{H^{m+2}(\Omega)} \leq \overline{C_{m}}\left(\|\theta\|_{L^{2}(\Omega)}+\|\bar{g}\|_{H^{m}(\Omega)}\right)
$$

\section{A Prior Estimates for Higher Regularity}

In this part, we need the following prior estimates to prove the local existence of the solution. Assume that the following inequalities hold:

$$
\begin{gathered}
\left\|\left(u_{0}, \theta_{0}\right)_{H^{2}}\right\|+1 \leq C_{0} \\
\sup _{0 \leq t \leq T_{*}}\left(\|u(t)\|_{H^{2}}+\left\|u_{t}(t)\right\|_{L^{2}}\right)+\int_{0}^{T_{*}}\left(\left\|\nabla u_{t}\right\|_{L^{2}}^{2}+\left\|\nabla^{2} u\right\|_{L^{q}}^{2}\right) \mathrm{d} t+1 \leq C_{1}
\end{gathered}
$$

where $1 \leq C_{0} \leq C_{1}, \quad 0<T_{*} \leq T$.

Remark: $C$ is a constant if be not added. 


\subsection{Estimate for Temperature}

Lemma 3.1.1. $\|\theta\|_{L^{2}}^{2}+\int_{0}^{t}\|\nabla \theta\|_{L^{2}}^{2} \mathrm{~d} s \leq C$

Proof: Multiplying the second equation of (1.1) by $\theta$ and integrating over $\Omega$, one has

$$
\begin{gathered}
\frac{1}{2} \frac{\mathrm{d}}{\mathrm{d} t} \int_{\Omega} \theta^{2} \mathrm{~d} x=\int_{\Omega} \varepsilon \Delta \theta \cdot \theta-(u \cdot \nabla) \theta \cdot \theta \mathrm{d} x \\
\int_{\Omega} \varepsilon \Delta \theta \cdot \theta \mathrm{d} x \leq C\left(\int_{\partial \Omega} \frac{\partial \theta}{\partial n} \theta \mathrm{d} s-\int_{\Omega}(\nabla \theta)^{2} \mathrm{~d} x\right) \\
=C\left(\int_{\partial \Omega} \alpha \theta^{2} \mathrm{~d} s-\int_{\Omega}(\nabla \theta)^{2} \mathrm{~d} x\right) \\
\leq C \int_{\Omega}\left(\theta^{2}+|\theta| \cdot|\nabla \theta|\right) \mathrm{d} x+C \int_{\Omega}(\nabla \theta)^{2} \mathrm{~d} x \\
\leq C\left(\|\theta\|_{L^{2}}+\|\nabla \theta\|_{L^{2}}+C_{\delta}\|\theta\|_{L^{2}}+\delta\|\nabla \theta\|_{L^{2}}\right) \\
\int_{\Omega}(u \cdot \nabla) \theta \cdot \theta \mathrm{d} x=\int_{\Omega} u \cdot \nabla \theta \cdot \theta \mathrm{d} x \leq\|u\|_{L^{2}}\|\theta\|_{H^{1}}\|\nabla \theta\|_{L^{2}} \\
\leq\|u\|_{L^{2}}^{\frac{1}{2}}\|u\|_{H^{1}}^{\frac{1}{2}}\left(\|\theta\|_{L^{2}}+\|\nabla \theta\|_{L^{2}}\right)\|\nabla \theta\|_{L^{2}} \\
\leq C\left(\|\theta\|_{L^{2}}\|\nabla \theta\|_{L^{2}}+\|\nabla \theta\|_{L^{2}}^{2}\right) \\
\leq C\left(\delta\|\nabla \theta\|_{L^{2}}^{2}+C_{\delta}\|\theta\|_{L^{2}}^{2}+\|\nabla \theta\|_{L^{2}}^{2}\right)
\end{gathered}
$$

Substituting (3.1.2) - (3.1.3) into (3.1.1), letting $\delta$ small enough and using Gronwall's inequality:

$$
\|\theta\|_{L^{2}}^{2}+\int_{0}^{t}\|\nabla \theta\|_{L^{2}}^{2} \mathrm{~d} s \leq C
$$

Lemma 3.1.2. $\|\nabla \theta\|_{L^{2}}^{2}+\int_{0}^{t}\left\|\theta_{t}\right\|_{L^{2}}^{2} \mathrm{~d} s \leq C$

Proof: Multiplying the second equation of (1.1) by $\theta_{t}$ and integrating over $\Omega$, one has

$$
\begin{gathered}
\int_{\Omega} \theta_{t}^{2} \mathrm{~d} x=\int_{\Omega} \varepsilon \Delta \theta \cdot \theta_{t}-(u \cdot \nabla) \theta \cdot \theta_{t} \mathrm{~d} x \\
\int_{\Omega} \varepsilon \Delta \theta \cdot \theta_{t} \mathrm{~d} x=\int_{\partial \Omega} \varepsilon \frac{\partial \theta}{\partial n} \theta_{t} \mathrm{~d} s-\int_{\Omega} \varepsilon \nabla \theta \cdot \nabla \theta_{t} \mathrm{~d} x \\
=\frac{1}{2} \frac{\mathrm{d}}{\mathrm{d} t} \int_{\partial \Omega} \varepsilon \alpha \theta^{2} \mathrm{~d} s-\frac{1}{2} \frac{\mathrm{d}}{\mathrm{d} t} \int_{\Omega} \varepsilon(\nabla \theta)^{2} \mathrm{~d} x \\
\leq C \frac{\mathrm{d}}{\mathrm{d} t} \int_{\Omega}\left(|\theta|^{2}+|\theta| \cdot|\nabla \theta|\right)+(\nabla \theta)^{2} \mathrm{~d} x \\
\leq C \frac{\mathrm{d}}{\mathrm{d} t}\left(C_{\delta}\|\nabla \theta\|_{L^{2}}^{2}+\delta\|\theta\|_{L^{2}}^{2}+\|\nabla \theta\|_{L^{2}}^{2}\right) \\
\int_{\Omega}(u \cdot \nabla) \theta \cdot \theta_{t} \mathrm{~d} x=\int_{\Omega} u \cdot \nabla \theta \cdot \theta_{t} \mathrm{~d} x \leq\|u\|_{L^{\infty}}\left\|\theta_{t}\right\|_{L^{2}}\|\nabla \theta\|_{L^{2}} \\
\leq C\left(\delta\left\|\theta_{t}\right\|_{L^{2}}^{2}+C_{\delta}\|\nabla \theta\|_{L^{2}}^{2}\right)
\end{gathered}
$$

Substituting (3.1.5) - (3.1.6) into (3.1.4), letting $\delta$ small enough and using Gronwall's inequality,

$$
\|\nabla \theta\|_{L^{2}}^{2}+\int_{0}^{t}\left\|\theta_{t}\right\|_{L^{2}}^{2} \mathrm{~d} s \leq C
$$

Lemma 3.1.3. $\left\|\theta_{t}\right\|_{L^{2}}^{2}+\int_{0}^{t}\left\|\nabla \theta_{t}\right\|_{L^{2}}^{2} \mathrm{~d} s \leq C$ 
Proof: Differentiating the second equation of (1.1) with respect to $t$, multiplying the second equation of (1.1) by $\theta_{t}$ and integrating over $\Omega$, one has

$$
\begin{aligned}
& \frac{1}{2} \frac{\mathrm{d}}{\mathrm{d} t} \int_{\Omega} \theta_{t}^{2} \mathrm{~d} x=\int_{\Omega} \varepsilon \Delta \theta_{t} \cdot \theta_{t}-u_{t} \cdot \nabla \theta \cdot \theta_{t}-u \cdot \nabla \theta_{t} \cdot \theta_{t} \mathrm{~d} x \\
& \int_{\Omega} \varepsilon \Delta \theta_{t} \cdot \theta_{t} \mathrm{~d} x=\int_{\partial \Omega} \varepsilon \frac{\partial \theta_{t}}{\partial n} \theta_{t} \mathrm{~d} s-\int_{\Omega} \varepsilon\left(\nabla \theta_{t}\right)^{2} \mathrm{~d} x \\
& \leq C\left(\int_{\partial \Omega} \theta_{t}^{2} \mathrm{~d} s-\int_{\Omega}\left(\nabla \theta_{t}\right)^{2} \mathrm{~d} x\right) \\
& \leq C\left(\int_{\Omega} \theta_{t}^{2}+\nabla \theta_{t} \cdot \theta_{t} \mathrm{~d} x+\left\|\nabla \theta_{t}\right\|_{L^{2}}^{2}\right) \\
& \leq C\left(\left\|\theta_{t}\right\|_{L^{2}}^{2}+\left\|\nabla \theta_{t}\right\|_{L^{2}}\left\|\theta_{t}\right\|_{L^{2}}+\left\|\nabla \theta_{t}\right\|_{L^{2}}^{2}\right) \\
& \leq C\left(\left\|\theta_{t}\right\|_{L^{2}}^{2}+C_{\delta}\left\|\nabla \theta_{t}\right\|_{L^{2}}^{2}+\delta\left\|\theta_{t}\right\|_{L^{2}}^{2}+\left\|\nabla \theta_{t}\right\|_{L^{2}}^{2}\right) \\
& \int_{\Omega} u_{t} \cdot \nabla \theta \cdot \theta_{t} \mathrm{~d} x \leq\left\|u_{t}\right\|_{L^{3}}\|\nabla \theta\|_{L^{2}}\left\|\theta_{t}\right\|_{H^{1}} \leq C\left\|u_{t}\right\|_{L^{2}}^{\frac{1}{2}}\|\theta\|_{L^{6}}^{\frac{1}{2}}\left\|\theta_{t}\right\|_{H^{1}} \\
& \leq C\left(C_{\delta}\left\|\theta_{t}\right\|_{L^{2}}^{2}+\delta\left\|u_{t}\right\|_{L^{2}}\|\theta\|_{H^{1}}+C_{\kappa}\left\|\nabla \theta_{t}\right\|_{L^{2}}^{2}+\kappa\left\|u_{t}\right\|_{L^{2}}\|\theta\|_{H^{1}}\right) \\
& \int_{\Omega} u \cdot \nabla \theta_{t} \cdot \theta_{t} \mathrm{~d} x \leq\|u\|_{L^{\infty}}\left\|\theta_{t}\right\|_{L^{2}}\left\|\nabla \theta_{t}\right\|_{L^{2}} \leq C_{\delta}\left\|\theta_{t}\right\|_{L^{2}}^{2}+\delta\left\|\nabla \theta_{t}\right\|_{L^{2}}^{2}
\end{aligned}
$$

Substituting (3.1.8) - (3.1.10) into (3.1.7), letting $\delta$ and $\kappa$ small enough and using Gronwall's inequality,

$$
\left\|\theta_{t}\right\|_{L^{2}}^{2}+\int_{0}^{t}\left\|\nabla \theta_{t}\right\|_{L^{2}}^{2} \mathrm{~d} s \leq C
$$

\subsection{Estimate for Velocity}

Lemma 3.2.1. $\|u\|_{L^{2}}^{2}+\int_{0}^{t}\|\nabla u\|_{L^{2}}^{2} \mathrm{~d} s \leq C$

Proof: Multiplying the first equation of (1.1) by $u$ and integrating over $\Omega$, one has

$$
\begin{gathered}
\frac{1}{2} \frac{\mathrm{d}}{\mathrm{d} t} \int_{\Omega} u^{2} \mathrm{~d} x=\int_{\Omega} \gamma \Delta u \cdot u+u^{2} \cdot \nabla u-\nabla p \cdot u+\theta f \cdot u \mathrm{~d} x \\
\int_{\Omega} \gamma \Delta u \cdot u \mathrm{~d} x=\int_{\Omega} 2 \gamma \operatorname{div}(S u) \cdot u \mathrm{~d} x=2 \gamma\left(\int_{\partial \Omega} S u \cdot n \cdot u \mathrm{~d} s-\int_{\Omega} S u \cdot \nabla u \mathrm{~d} x\right) \\
\leq C\left(\int_{\partial \Omega} B u \cdot u \mathrm{~d} s+\int_{\Omega} S u \cdot \nabla u \mathrm{~d} x\right) \leq C\left(\|u\|_{L^{2}}^{2}+\|\nabla u\|_{L^{2}}^{2}\right) \\
\int_{\Omega}(u \cdot \nabla) u \cdot u \mathrm{~d} x=\int_{\Omega} u^{2} \cdot \nabla u \mathrm{~d} x \leq\|u\|_{L^{3}}\|u\|_{H^{1}}\|\nabla u\|_{L^{2}} \\
\leq\|u\|_{L^{2}}^{\frac{1}{2}}\|u\|_{H^{1}}^{1}\left(\|u\|_{L^{2}}+\|\nabla u\|_{L^{2}}\right)\|\nabla u\|_{L^{2}} \\
\leq C\left(\|u\|_{L^{2}}\|\nabla u\|_{L^{2}}+\|\nabla u\|_{L^{2}}^{2}\right) \\
\leq C\left(\delta\|\nabla u\|_{L^{2}}^{2}+C_{\delta}\|u\|_{L^{2}}^{2}+\|\nabla u\|_{L^{2}}^{2}\right) \\
\int_{\Omega} \nabla p \cdot u \mathrm{~d} x=\|\nabla p\|_{L^{2}}\|u\|_{L^{2}} \leq C_{\delta}\|u\|_{L^{2}}^{2}+\delta\|\nabla p\|_{L^{2}}^{2} \\
\int_{\Omega} \theta f \cdot u \mathrm{~d} x=\|\theta\|_{L^{2}}\|f\|_{L^{\infty}}\|u\|_{L^{2}} \leq C\left(C_{\delta}\|u\|_{L^{2}}^{2}+\delta\|\theta\|_{L^{2}}^{2}\right)
\end{gathered}
$$

Substituting (3.2.2) - (3.2.5) into (3.2.1), letting $\delta$ small enough and using Gronwall's inequality, 


$$
\|u\|_{L^{2}}^{2}+\int_{0}^{t}\|\nabla u\|_{L^{2}}^{2} \mathrm{~d} s \leq C
$$

Lemma 3.2.2. $\|\nabla u\|_{L^{2}}^{2}+\int_{0}^{t}\left\|u_{t}\right\|_{L^{2}}^{2} \mathrm{~d} s \leq C$

Proof: Multiplying the first equation of (1.1) by $u_{t}$ and integrating over $\Omega$, one has

$$
\begin{gathered}
\int_{\Omega} u_{t}^{2} \mathrm{~d} x=\int_{\Omega} \gamma \Delta u \cdot u_{t}+u \cdot \nabla u \cdot u_{t}-\nabla p \cdot u_{t}+\theta f \cdot u_{t} \mathrm{~d} x \\
\int_{\Omega} \gamma \Delta u \cdot u_{t} \mathrm{~d} x=\int_{\Omega} 2 \gamma d i v(S u) \cdot u_{t} \mathrm{~d} x=2 \gamma\left(\int_{\partial \Omega} S u \cdot n \cdot u_{t} \mathrm{~d} s-\int_{\Omega} S u \cdot \nabla u_{t} \mathrm{~d} x\right) \\
\leq C\left(\int_{\partial \Omega} B u \cdot u_{t} \mathrm{~d} s+\int_{\Omega} S u \cdot \nabla u_{t} \mathrm{~d} x\right) \leq C \frac{\mathrm{d}}{\mathrm{d} t}\left(\|u\|_{L^{2}}^{2}+\|\nabla u\|_{L^{2}}^{2}\right) \\
\int_{\Omega}(u \cdot \nabla) u \cdot u_{t} \mathrm{~d} x=\int_{\Omega} u \cdot \nabla u \cdot u_{t} \mathrm{~d} x \leq\|u\|_{L^{\infty}}\left\|u_{t}\right\|_{L^{2}}\|\nabla u\|_{L^{2}} \\
\leq C\left(C_{\delta}\left\|u_{t}\right\|_{L^{2}}^{2}+\delta\|\nabla u\|_{L^{2}}^{2}\right) \\
\int_{\Omega} \nabla p \cdot u_{t} \mathrm{~d} x=\|\nabla p\|_{L^{2}}\left\|u_{t}\right\|_{L^{2}} \leq C_{\delta}\left\|u_{t}\right\|_{L^{2}}^{2}+\delta\|\nabla p\|_{L^{2}}^{2} \\
\int_{\Omega} \theta f \cdot u_{t} d x=\|\theta\|_{L^{2}}\|f\|_{L^{\infty}}\left\|u_{t}\right\|_{L^{2}} \leq C\left(C_{\delta}\left\|u_{t}\right\|_{L^{2}}^{2}+\delta\|\theta\|_{L^{2}}^{2}\right)
\end{gathered}
$$

Substituting (3.2.7) - (3.2.10) into (3.2.6), letting $\delta$ small enough and using Gronwall's inequality,

$$
\|\nabla u\|_{L^{2}}^{2}+\int_{0}^{t}\left\|u_{t}\right\|_{L^{2}}^{2} \mathrm{~d} s \leq C
$$

Lemma 3.2.3. $\left\|u_{t}\right\|_{L^{2}}^{2}+\int_{0}^{t}\left\|\nabla u_{t}\right\|_{L^{2}}^{2} \mathrm{~d} s \leq C$

Proof: Differentiating the first equation of (1.1) with respect to $t$, multiplying the first equation of (1.1) by $u_{t}$ and integrating over $\Omega$, one has

$$
\begin{gathered}
\int_{\Omega} u_{t} u_{t} \mathrm{~d} x \\
=\int_{\Omega} \gamma \Delta u_{t} \cdot u_{t}+u_{t} \cdot \nabla u \cdot u_{t}+u \cdot \nabla u_{t} \cdot u_{t}-\nabla p_{t} \cdot u_{t}+\theta_{t} f \cdot u_{t}+\theta f_{t} \cdot u_{t} \mathrm{~d} x \\
\int_{\Omega} \gamma \Delta u_{t} \cdot u_{t} \mathrm{~d} x=\int_{\Omega} 2 \gamma \operatorname{div}\left(S u_{t}\right) \cdot u_{t} \mathrm{~d} x=2 \gamma\left(\int_{\partial \Omega} S u_{t} \cdot n \cdot u_{t} \mathrm{~d} s-\int_{\Omega} S u_{t} \cdot \nabla u_{t} \mathrm{~d} x\right) \\
\leq C\left(\int_{\partial \Omega} B u_{t} \cdot u_{t} \mathrm{~d} s+\int_{\Omega} S u_{t} \cdot \nabla u_{t} \mathrm{~d} x\right) \leq C\left(\left\|u_{t}\right\|_{L^{2}}^{2}+\left\|\nabla u_{t}\right\|_{L^{2}}^{2}\right) \\
\int_{\Omega} u_{t} \cdot \nabla u \cdot u_{t} \mathrm{~d} x \leq\left\|u_{t}\right\|_{L^{3}}\|\nabla u\|_{L^{2}}\left\|u_{t}\right\|_{H^{1}} \leq C\left\|u_{t}\right\|_{L^{2}}^{\frac{1}{2}}\left\|u_{t}\right\|_{L^{6}}^{\frac{1}{2}}\left\|u_{t}\right\|_{H^{1}} \\
\leq C\left(C_{\delta}\left\|u_{t}\right\|_{L^{2}}^{2}+\delta\left\|u_{t}\right\|_{L^{2}}\left\|u_{t}\right\|_{H^{1}}+C_{\kappa}\left\|\nabla u_{t}\right\|_{L^{2}}^{2}+\kappa\left\|u_{t}\right\|_{L^{2}}\left\|u_{t}\right\|_{H^{1}}\right) \\
\int_{\Omega} u \cdot \nabla u_{t} \cdot u_{t} \mathrm{~d} x \leq\|u\|_{L^{\infty}}\left\|u_{t}\right\|_{L^{2}}\left\|\nabla u_{t}\right\|_{L^{2}} \leq C_{\delta}\left\|u_{t}\right\|_{L^{2}}^{2}+\delta\left\|\nabla u_{t}\right\|_{L^{2}}^{2} \\
\int_{\Omega} \nabla p_{t} \cdot u_{t} \mathrm{~d} x=\left\|\nabla p_{t}\right\|_{L^{2}}\left\|u_{t}\right\|_{L^{2}} \leq C_{\delta}\left\|u_{t}\right\|_{L^{2}}^{2}+\delta\left\|\nabla p_{t}\right\|_{L^{2}}^{2} \\
\int_{\Omega} \theta t f \cdot u_{t} \mathrm{~d} x=\left\|\theta_{t}\right\|_{L^{2}}\|f\|_{L^{\infty}}\left\|u_{t}\right\|_{L^{2}} \leq C\left(C_{\delta}\left\|u_{t}\right\|_{L^{2}}^{2}+\delta\left\|\theta_{t}\right\|_{L^{2}}^{2}\right) \\
\int_{\Omega} \theta f_{t} \cdot u_{t} \mathrm{~d} x=\|\theta\|_{L^{2}}\left\|f_{t}\right\|_{L^{\infty}}\left\|u_{t}\right\|_{L^{2}} \leq C\left(C_{\delta}\left\|u_{t}\right\|_{L^{2}}^{2}+\delta\|\theta\|_{L^{2}}^{2}\right)
\end{gathered}
$$

Substituting (3.2.12)-(3.2.17) into (3.2.11), letting $\delta$ and $\kappa$ small enough and using Gronwall's inequality,

$$
\left\|u_{t}\right\|_{L^{2}}^{2}+\int_{0}^{t}\left\|\nabla u_{t}\right\|_{L^{2}}^{2} \mathrm{~d} s \leq C
$$




\subsection{Elliptic Estimates for Velocity and Temperature}

Lemma 3.3.1. $\|\nabla \theta\|_{H^{1}} \leq C$

Proof: According to the second equation of (1.1), we have $-\Delta \theta=-\frac{\theta_{t}}{\varepsilon}-\frac{(u \cdot \nabla) \theta}{\varepsilon}$, then use the elliptic regularity:

$$
\begin{gathered}
\|\theta\|_{H^{2}} \leq C\left(\left\|-\theta_{t}-(u \cdot \nabla) \theta\right\|_{L^{2}}+\|\theta\|_{L^{2}}\right) \leq C\left(\left\|\theta_{t}\right\|_{L^{2}}+\|\nabla \theta \cdot u\|_{L^{2}}+\|\theta\|_{L^{2}}\right) \\
\|\nabla \theta\|_{H^{1}} \leq\|\theta\|_{H^{2}} \leq C\left(\left\|\theta_{t}\right\|_{L^{2}}+\|\nabla \theta \cdot u\|_{L^{2}}+\|\theta\|_{L^{2}}\right) \\
\leq C\left(\left\|\theta_{t}\right\|_{L^{2}}+\|u\|_{L^{\infty}}\|\nabla \theta\|_{L^{2}}+\|\theta\|_{L^{2}}\right) \leq C
\end{gathered}
$$

Lemma 3.3.2. $\int_{0}^{t}\|\nabla \theta\|_{W^{1, q}}^{2} \mathrm{~d} s \leq C$

Proof: According to the second equation of (1.1), we have $-\Delta \theta=-\frac{\theta_{t}}{\varepsilon}-\frac{(u \cdot \nabla) \theta}{\varepsilon}$, then use the elliptic regularity:

$$
\|\theta\|_{W^{2, q}} \leq C\left(\left\|-\theta_{t}-(u \cdot \nabla) \theta\right\|_{L^{q}}+\|\theta\|_{L^{q}}\right) \leq C\left(\left\|\theta_{t}\right\|_{L^{q}}+\|\nabla \theta \cdot u\|_{L^{q}}+\|\theta\|_{L^{q}}\right)
$$

Both sides of the above inequality multiply by itself, then

$$
\begin{gathered}
\|\theta\|_{W^{2, q}}^{2} \leq C\left(\left\|\theta_{t}\right\|_{L^{q}}^{2}+\|\nabla \theta \cdot u\|_{L^{q}}^{2}+\|\theta\|_{L^{q}}^{2}\right) \\
\|\nabla \theta\|_{W^{1, q}}^{2} \leq\|\theta\|_{W^{2, q}}^{2} \leq C\left(\left\|\theta_{t}\right\|_{L^{q}}^{2}+\|\nabla \theta \cdot u\|_{L^{q}}^{2}+\|\theta\|_{L^{q}}^{2}\right) \\
\leq C\left(\left\|\theta_{t}\right\|_{H^{1}}^{2}+\|u\|_{L^{\infty}}^{2}\|\nabla \theta\|_{H^{1}}^{2}+\|\theta\|_{H^{1}}^{2}\right)
\end{gathered}
$$

And integrating over $(0, t)$, we have

$$
\int_{0}^{t}\|\nabla \theta\|_{W^{1, q}}^{2} \mathrm{~d} s \leq \int_{0}^{t} C\left(\left\|\theta_{t}\right\|_{H^{1}}^{2}+\|u\|_{L^{\infty}}^{2}\|\nabla \theta\|_{H^{1}}^{2}+\|\theta\|_{H^{1}}^{2}\right) \mathrm{d} s \leq C
$$

Lemma 3.3.3. $\|\nabla u\|_{H^{1}} \leq C$

Proof: According to the first equation of (1.1), we have $-\Delta u=-\frac{u_{t}}{\gamma}-\frac{(u \cdot \nabla) u}{\gamma}-\frac{\nabla p}{\gamma}+\frac{\theta f}{\gamma}$, then use the elliptic regularity:

$$
\begin{gathered}
\|u\|_{H^{2}} \leq C\left(\left\|-u_{t}-(u \cdot \nabla) u-\nabla p+\theta f\right\|_{L^{2}}+\|u\|_{L^{2}}\right) \\
\leq C\left(\left\|u_{t}\right\|_{L^{2}}+\|(u \cdot \nabla) u\|_{L^{2}}+\|\nabla p\|_{L^{2}}+\|\theta f\|_{L^{2}}+\|u\|_{L^{2}}\right) \\
\|\nabla u\|\left\|_{H^{1}} \leq\right\| u \|_{H^{2}} \leq C\left(\left\|u_{t}\right\|_{L^{2}}+\|(u \cdot \nabla) u\|_{L^{2}}+\|\nabla p\|_{L^{2}}+\|\theta f\|_{L^{2}}+\|u\|_{L^{2}}\right) \\
\leq C\left(\left\|u_{t}\right\|_{L^{2}}+\|u\|_{L^{2}}\|\nabla u\|_{H^{1}}+\|\nabla p\|_{L^{2}}+\|f\|_{L^{\infty}}\|\theta\|_{L^{2}}+\|u\|_{L^{2}}\right) \\
\leq C\left(\left\|u_{t}\right\|_{L^{2}}+\|u\|_{L^{2}}^{\frac{1}{2}}\|u\|_{H^{1}}^{\frac{1}{2}}\|\nabla u\|_{H^{1}}+\|\nabla p\|_{L^{2}}+\|f\|_{L^{\infty}}\|\theta\|_{L^{2}}+\|u\|_{L^{2}}\right) \\
\leq C
\end{gathered}
$$

Lemma 3.3.4. $\int_{0}^{t}\|\nabla u\|_{W^{1, q}}^{2} \mathrm{~d} s \leq C$

Proof: According to the first equation of (1.1), we have $-\Delta u=-\frac{u_{t}}{\gamma}-\frac{(u \cdot \nabla) u}{\gamma}-\frac{\nabla p}{\gamma}+\frac{\theta f}{\gamma}$, then use the elliptic regularity: 


$$
\begin{aligned}
\|u\|_{W^{2, q}} & \leq C\left(\left\|-u_{t}-(u \cdot \nabla) u-\nabla p+\theta f\right\|_{L^{q}}+\|u\|_{L^{q}}\right) \\
& \leq C\left(\left\|u_{t}\right\|_{L^{q}}+\|(u \cdot \nabla) u\|_{L^{q}}+\|\nabla p\|_{L^{q}}+\|\theta f\|_{L^{q}}+\|u\|_{L^{q}}\right)
\end{aligned}
$$

Both sides of the above inequality multiply by itself, then

$$
\begin{gathered}
\|u\|_{W^{2, q}}^{2} \leq C\left(\left\|u_{t}\right\|_{L^{q}}^{2}+\|(u \cdot \nabla) u\|_{L^{q}}^{2}+\|\nabla p\|_{L^{q}}^{2}+\|\theta f\|_{L^{q}}^{2}+\|u\|_{L^{q}}^{2}\right) \\
\|\nabla u\|_{W^{1, q}}^{2} \leq\|u\|_{W^{2, q}}^{2} \leq C\left(\left\|u_{t}\right\|_{L^{q}}^{2}+\|(u \cdot \nabla) u\|_{L^{q}}^{2}+\|\nabla p\|_{L^{q}}^{2}+\|\theta f\|_{L^{q}}^{2}+\|u\|_{L^{q}}^{2}\right) \\
\leq C\left(\left\|u_{t}\right\|_{H^{1}}^{2}+\|u\|_{L^{\infty}}^{2}\|\nabla u\|_{H^{1}}^{2}+\|\nabla p\|_{L^{q}}^{2}+\|f\|_{L^{\infty}}^{2}\|\theta\|_{H^{1}}^{2}+\|u\|_{H^{1}}^{2}\right)
\end{gathered}
$$

And integrating over $(0, t)$, we have $\int_{0}^{t}\|\nabla u\|_{W^{1, q}}^{2} \mathrm{~d} s \leq C$.

\section{The Local Existence of the Solution of Boussinesq Equations}

First, we consider the following linearized system:

$$
\left\{\begin{array}{l}
u_{t}+(v \cdot \nabla) u+\nabla p=\gamma \Delta u+\theta f, \\
\theta_{t}+(u \cdot \nabla) \theta=\varepsilon \Delta \theta, \\
\operatorname{div} u=0,
\end{array} \quad(x, t) \in \Omega \times(0, t)\right.
$$

Lemma 4.1. Let $\Omega$ be a bounded domain in $R^{3}$ with smooth boundary, when $3<q \leq 6$, we have $\left(u_{0}, \theta_{0}\right) \in H^{1}(\Omega)$. Assume that

$$
\begin{gathered}
v \in L^{\infty}\left([0, T] ; H^{2}(\Omega)\right) \cap L^{2}\left([0, T] ; W^{2, q}(\Omega)\right), \\
v_{t} \in L^{\infty}\left([0, T] ; L^{2}\right) \cap L^{2}\left([0, T] ; H^{1}\right)
\end{gathered}
$$

with the boundary conditions:

$$
\left.v \cdot n\right|_{\partial \Omega}=0,\left.(S v \cdot n)_{\tau}\right|_{\partial \Omega}=(B v)_{\tau}
$$

Then there is a unique strong solution $(u, \theta)$ meeting (1.1) - (1.3) such tat

$$
\begin{aligned}
& (u, \theta) \in C\left([0, T] ; H^{2}(\Omega)\right) \cap L^{2}\left([0, T] ; W^{2, q}(\Omega)\right) ; \\
& \left(u_{t}, \theta_{t}\right) \in C\left([0, T] ; L^{2}(\Omega)\right) \cap L^{2}\left([0, T] ; H^{1}(\Omega)\right)
\end{aligned}
$$

Proof: It follows from Theorem 4 in chapter 5.9 [22], then we obtain $v \in C\left([0, T] ; H^{1}\right)$.

Next, Gerlakin approach is applied to prove the local existence of the solution of Equation (4.1).

Assume that $\left\{\phi_{l}\right\}_{1}^{m}$ and $\left\{\omega_{l}\right\}_{1}^{m}$ respectively representing the eigenvectors of the operator $-\Delta$ and the operator $L$ are smooth functions.

$$
V^{m}=\operatorname{span}\left\{\phi_{1}, \phi_{2}, \cdots, \phi_{m}\right\}, W^{m}=\operatorname{span}\left\{\omega_{1}, \omega_{2}, \cdots, \omega_{m}\right\}
$$

for a positive constant $m$ fixed, let

$$
\theta_{m}(t)=\sum_{l=1}^{m} a_{m}^{l}(t) \phi_{l}, \quad u_{m}(t)=\sum_{l=1}^{m} b_{m}^{l}(t) \omega_{l}
$$

We hope that the coefficients $a_{m}^{l}(t), b_{m}^{l}(t)$ satisfy: 


$$
\left\{\begin{array}{l}
\int_{\Omega} u_{t m} \omega_{m}+v_{m} \nabla u_{m} u_{t m} \omega_{m}+\nabla p_{m} u_{t m} \omega_{m} \mathrm{~d} x=\int_{\Omega} \gamma \Delta u_{m} u_{t m} \omega_{m}+\theta_{m} f_{m} u_{t m} \omega_{m} \mathrm{~d} x \\
\int_{\Omega} \theta_{t m} \phi_{m}+u_{m} \nabla \theta_{m} \phi_{m} \mathrm{~d} x=\int_{\Omega} \varepsilon \Delta \theta_{m} \phi_{m} \mathrm{~d} x
\end{array}\right.
$$

Thus we seek functions $\left(u_{m}, \theta_{m}\right)$ that satisfy the "projection" (4.4) of problem (4.1) onto the finite dimensional subspace spanned by $\left(\left\{\omega_{l}\right\}_{1}^{m},\left\{\phi_{l}\right\}_{1}^{m}\right)$.

It follows from Theorem 1 in chapter 7 [22], then we obtain that for $\forall m$, there exists unique $\left(u_{m}, \theta_{m}\right)$ satisfying Equation (4.4).

\section{The Proof of Theme 1.2}

Similar to the prior estimates in part 3, we have

$$
\sup _{0 \leq t \leq T_{*}}\left(\left\|u_{m}(t)\right\|_{H^{2}}+\left\|u_{t m}(t)\right\|_{L^{2}}\right)+\int_{0}^{T_{*}}\left(\left\|\nabla u_{t m}\right\|_{L^{2}}^{2}+\left\|\nabla^{2} u_{m}\right\|_{L^{q}}^{2}\right) \mathrm{d} t+1 \leq C_{1}
$$

where $C$ has no connection with $m$, then we have

$$
\begin{gathered}
\left(u_{m}, \theta_{m}\right) \rightarrow(u, \theta) \text { in } L^{\infty}\left(0, T ; H^{1}\right) \\
\left(u_{m}, \theta_{m}\right) \stackrel{\text { weakly }}{\longrightarrow}(u, \theta) \text { in } L^{2}\left(0, T ; W^{2, q}\right) \\
\left(u_{m}, \theta_{m}\right) \stackrel{\text { weakly }}{\longrightarrow}(u, \theta) \text { in } L^{\infty}\left(0, T ; H^{2}\right) \\
\left(\partial_{t} u_{m}, \partial_{t} \theta_{m}\right) \stackrel{\text { weak }}{\longrightarrow}\left(u_{t}, \theta_{t}\right) \text { in } L^{2}\left(0, T ; H^{1}\right)
\end{gathered}
$$

It follows from Theorem 3 in chapter 7 [22], let $m \rightarrow \infty$ then we obtain that $(u, \theta)$ is the solution of (4.1).

The proof of Lemma 4.1 is completed.

Next, the iteration method is used to prove the local existence of the solution of Boussinesq equations.

Construct approximate solutions of Boussinesq equations that meet the initial and boundary problems (1.2) - (1.3).

1) define $u^{0}=0$,

2) assume that $k \geq 1$, define $v=u^{k-1}$,

$$
\left\{\begin{array}{l}
u_{t}^{k}+\left(u^{k-1} \cdot \nabla\right) u^{k}+\nabla p^{k}=\gamma \Delta u^{k}+\theta^{k} f^{k}, \\
\theta_{t}^{k}+\left(u^{k} \cdot \nabla\right) \theta^{k}=\varepsilon \Delta \theta^{k}, \\
\operatorname{div}^{k}=0,
\end{array} \quad(x, t) \in \Omega \times(0, t)\right.
$$

Initial conditions:

$$
\left.\left(u^{k}, \theta^{k}\right)\right|_{t=0}=\left(u_{0}^{k}, \theta_{0}^{k}\right)
$$

Boundary conditions:

$$
\left.u^{k} \cdot n\right|_{\partial \Omega}=0,\left.\frac{\partial \theta^{k}}{\partial n}\right|_{\partial \Omega}=\alpha \theta^{k},\left.\left(S u^{k} \cdot n\right)_{\tau}\right|_{\partial \Omega}=\left(B u^{k}\right)_{\tau}
$$

According to Lemma 4.1, we can know that the problems (5.1) - (5.3) exist the local solutions $\left(u^{k}, \theta^{k}\right)$. Furthermore, according to the prior estimates, we get

$$
\sup _{0 \leq t \leq T_{*}}\left(\left\|u^{k}\right\|_{H^{2}}+\left\|u_{t}^{k}\right\|_{L^{2}}\right)+\int_{0}^{T_{*}}\left(\left\|\nabla u_{t}^{k}\right\|_{L^{2}}^{2}+\left\|\nabla^{2} u^{k}\right\|_{L^{q}}^{2}\right) \mathrm{d} t+1 \leq C_{1}
$$

where $C$ has no connection with $k$. 
According to Aubin-Lions lemma, one has

$$
\begin{gathered}
\left(u^{k}, \theta^{k}\right) \rightarrow(u, \theta) \text { in } L^{\infty}\left(0, T_{*} ; H^{1}\right) \\
(u, \theta) \in L^{\infty}\left(\left[0, T_{*}\right] ; H^{2}(\Omega)\right) \cap L^{2}\left(\left[0, T_{*}\right] ; W^{2, q}(\Omega)\right) \\
\left(u_{t}, \theta_{t}\right) \in L^{\infty}\left(\left[0, T_{*}\right] ; L^{2}(\Omega)\right) \cap L^{2}\left(\left[0, T_{*}\right] ; H^{1}(\Omega)\right)
\end{gathered}
$$

Last, we show the continuity of $u$ and $\theta$ over time.

$$
\begin{aligned}
& \left\|\left(u_{t}\right)_{t}\right\|_{W^{*}}=\sup _{0 \leq t \leq T_{*}}\left|\left\langle\left(u_{t}\right)_{t}, \psi\right\rangle\right| \\
& \leq \sup _{0 \leq \leq \leq T_{*}} \int_{\Omega}\left|\gamma \Delta u_{t}+u_{t} \cdot \nabla u+u \cdot \nabla u_{t}+\nabla p_{t}+\theta_{t} f+\theta f_{t} \| \psi\right| \mathrm{d} x \\
& \leq \sup _{0 \leq t \leq T_{*}} C\left(\left(\left\|u_{t}\right\|_{L^{2}}\|\nabla u\|_{L^{3}}+\left\|\nabla u_{t}\right\|_{L^{2}}\|u\|_{L^{3}}+\left\|\theta_{t}\right\|_{L^{2}}\|f\|_{L^{3}}+\|\theta\|_{L^{2}}\left\|f_{t}\right\|_{L^{2}}\right)\|\psi\|_{H^{1}}\right. \\
& \left.\quad+\left\|\nabla p_{t}\right\|_{L^{2}}\|\psi\|_{L^{2}}+\left\|\nabla u_{t}\right\|_{L^{2}}\|\nabla \psi\|_{L^{2}}\right)+\sup _{0 \leq t \leq T_{*}} \int_{\partial \Omega} \nabla u_{t} \psi \mathrm{ds}
\end{aligned}
$$

where $W^{*}$ denotes the dual space of $W$. It follows from Theorem 3 in chapter 5.9 [22], then we obtain $u_{t} \in C\left(0, T_{*} ; L^{2}\right)$. Then according to the elliptic regularity, we have $\nabla^{2} u \in L^{\infty}\left(0, T_{*} ; L^{2}\right)$.

$$
\begin{aligned}
\left\|\left(\theta_{t}\right)_{t}\right\|_{W^{*}}= & \sup _{\|\varphi\|_{H^{1}=1}}\left|\left\langle\left(\theta_{t}\right)_{t}, \varphi\right\rangle\right| \\
\leq & \sup _{\|\varphi\|_{H^{1}}=1} \int_{\Omega}\left|\varepsilon \Delta \theta_{t}+u_{t} \cdot \nabla \theta+u \cdot \nabla \theta_{t} \| \varphi\right| \mathrm{d} x \\
\leq & \sup _{\|\varphi\|_{H^{1}}=1} C\left(\left(\left\|u_{t}\right\|_{L^{2}}\|\nabla \theta\|_{L^{3}}+\left\|\nabla \theta_{t}\right\|_{L^{2}}\|u\|_{L^{2}}\right)\|\varphi\|_{H^{1}}\right. \\
& \left.+\left\|\nabla \theta_{t}\right\|_{L^{2}}\|\nabla \varphi\|_{L^{2}}\right)+\sup _{\|\varphi\|_{H^{1}}=1} \int_{\partial \Omega} \nabla \theta_{t} \varphi \mathrm{d} s
\end{aligned}
$$

where $\left(H^{1}(\Omega)\right)^{*}$ denotes the dual space of $H^{1}(\Omega)$. This can show $\left(\theta_{t}\right)_{t} \in L^{2}\left(0, T_{*} ;\left(H^{1}(\Omega)\right)^{*}\right)$. So we have $\theta_{t} \in L^{2}\left(0, T_{*} ; H^{1}(\Omega)\right)$. Similar to the proof of the continuity of $u_{t}$, it is easy to know $\theta_{t} \in C\left(0, T_{*} ; L^{2}\right)$. Then according to the elliptic regularity, we have $\nabla^{2} \theta \in C\left(0, T_{*} ; L^{2}\right)$.

Above all, we complete the proof of the Theorem 1.2.

\section{References}

[1] Ye, Z., Xu, X.J. and Xue, L.T. (2017) On the Global Regularity of the 2D Boussinesq Equations with Fractional Dissipation. Mathematische Nachrichten, 290, 1420-1439. https://doi.org/10.1002/mana.201500413

[2] Ye, Z. and Xu, X.J. (2016) Global Well-Posedness of the 2D Boussinesq Equations with Fractional Laplacian Dissipation. Journal of Differential Equations, 260, 6716-6744. https://doi.org/10.1016/j.jde.2016.01.014

[3] Fan, J.S., Jia, X.J., Nakamura, G. and Zhou, Y. (2015) On Well-Posedness and Blowup Criteria for the Magnetohydrodynamics with the Hall and Ion-Slip Effects. $Z$. Angew. Math. Phys., 66, 1695-1706.

[4] Hmidi, T., Keraani, S. and Rousset, F. (2011) Global Well-Posedness for Euler-Boussinesq System with Critical Dissipation, Comm. Partial Differential Equa- 
tions, 36, 420-445. https://doi.org/10.1080/03605302.2010.518657

[5] Liu, Y. The Attenuation in $L^{2}$ of the Weak Existence of the Boussinesq Equations. Master's Thesis, Capital Normal University, Beijing.

[6] Li, W. (2008) The Exact Solutions of the Boussinesq Equations. Journal of Bohai University, 29.

[7] Li, X.J. The Global Stability in $L^{2}$ of the Boussinesq Equations in Three Dimensional Regional and Unbounded Regional. Master's Thesis, Capital Normal University, Beijing.

[8] Tan, Z. and Wang, Y.J. (2015) Global Well-Posedness of an Initial-Boundary Value Problem for Viscous Non-Resistive MHD Systems. Mathematics, arXiv: 1509.08349.

[9] Ohno, M. and Hirota, T. (1998) On the Initial-Boundary Value Problem for the Linearized MHD Equations. Sbrikaisekikenkybsho $K \wedge k y b r o k u, 1045,26-44$.

[10] Matsumura, and Nismda, T. (1983) Initial-Boundary Value Problems of the Equations of Motion of General Fluids. Comm. Math. Phys., 89, 445-446.

[11] LIONS (1998) Mathematical Topics in Fluid Mechanics. Vol. 2, Oxford Lecture Ser. Math. 10, Oxford University Press, New York.

[12] HOFF (2011) Existence of Solutions to a Model for Sparse, One-Dimensional Fluids. Journal of Differential Equations, 250, 1083-1113.

[13] HOFF and D.SERRE (1991) The Failure of Continuous Dependence on Initial Data for the Navier-Stokes Equations of Compressible Flow. SIAM Journal on Applied Mathematics, 51, 887-898. https://doi.org/10.1137/0151043

[14] HOFF (2005) Compressible Flow in a Half-Space with Navier Boundary Conditions. Journal of Mathematical Fluid Mechanics, 7, 315-338. https://doi.org/10.1007/s00021-004-0123-9

[15] HOFF (1997) Global Solutions of the Navier-Stokes Equations for Multidimensional, Compressible Flow with Discontinuous Initial Data. Journal of Differential Equations, 120, 1742-1760.

[16] Fan, J., Jiang, S., Nakamura, G. and Zhou, Y. (2011) Logarithmically Improved Regularity Criteria for the Navier-Stokes and MHD Equations. Journal of Mathematical Fluid Mechanics, 13, 557.

[17] HOFF and M.SANTOS (2008) Lagrangean Structure and Propagation of Singularities in Multidimensional Compressible Flow. Archive for Rational Mechanics and Analysis, 188, 509-543. https://doi.org/10.1007/s00205-007-0099-8

[18] HOFF (2006) Uniqueness of Weak Solutions of the Navier-Stokes Equations of Multidimensional Compressible Flow. SIAM Journal on Mathematical Analysis, 37, 1742-1760. https://doi.org/10.1137/040618059

[19] Hoff, D. (2012) Local Solutions of a Compressible Flow Problem with Navier Boundary Conditions in General Three Dimensional Domains Department of Mathematics. Indiana University, Bloomington, 633-650.

[20] Hmidi, T., Keraani, S. and Rousset, F. (2010) Global Well-Posedness for a Boussinesq-Navier-Stokes System with Critical Dissipation. Journal of Differential Equations, 249, 2147-2174.

[21] Hoff, D. (2011) Local Solutions of a Compressible Flow Problem with Navier Boundary Conditions in General Three-Dimensional Domains. SIAM Journal on Mathematical Analysis, 44, 1872-1886.

[22] Evans (2010) Partial Differential Equations, Grad. Texts in Math. 19, American Mathematical Society, Providence. 